\title{
9 The apparent realism of naïve realism How really naïve is naïve realism?
}

What I have been maintaining then is that our models, frames, logical and mathematical formulations about structure and our drawings and frames in the sciences and technologies are always originally created and interpreted technically by proposals that start by using terms from our ordinary language. We seem enamored by the wonderful complexity of our scientific models and we feel much the same with the enormous developments in mathematics that underlines them. We are so enamored and in awe of so much of it that many think that this complexity can only be intuitively grasped and understood from some notion of preexisting mental conditions from eternal forms such as we find in the writing of Plato and Aristotle. Some want to maintain that they are creations of the gods and that they are not a product of human artifice and construction.

I take this Platonic position of forms and essences and its philosophical jargon to be what is called 'ontological realism'. This view of eternal forms is to be contrasted with empirical pursuits of knowing where the basic features of reality are to be discovered in and through the sciences, especially in physics and astronomy, where the cognitive aim is to know about stuff and its patterns and arrangement and how it actually is spread out across the universe. The philosophical jargon for this rational quest for the structure of being that is sought in what is external to us through the senses is called 'epistemic realism'. Ontological realism posits forms as an object of thinking. Epistemic realism posits forms as an object of perception through the senses.

Sometimes the ideal modeled constructions of being and its structures seem so beautiful and elegant that we think that their beauty is truth, and their truth beauty, and that all that you need to know are the first principle that determined what is actually, ultimately and an absolutely real. Note the above strings of intensifying adverbs actually, ultimately, and absolutely seem to add nothing more of reality to anything of which we speak than when we simply speak of something as simply just real. But yet metaphysicians have maintained that there are different realms of being in the one and only singular universal being. Or again they often speak of reality where some things are more real than other things that are also thought to be real. Note some speak of degrees of reality as if whatever is real can be real as a matter of degree. It is by such play on the grammar of the term 'real', using it as a comparative adjective in characterizing multiple-categories and multiple-orders and rankings that some are able to create a plurality of different realities.

Realists thus in speaking of what is real comparatively are able to multiply the number of the levels of different forms of being as for example the 31 realms of being that are to be found in one version of Buddhist cosmology. Historically it has been no wonder that so many magical religious myths have gotten themselves entangled 
into such metaphysical speculations about the ground and structure of Being or in what is ultimately called the highest reality 'The One'. They seem in their religious quest interested in what is essentially prior even to God. It is in this quest for ultimate foundations for truth that dominates those who seek God by such faith in their traditional religious myths.

Note how thoughts of cosmology require conceptions of ontology and visa versa. As technically defined 'ontology' and 'cosmology' are correlative terms used and interpreted in speculative philosophy about realistic philosophical world views. It is an amazing speculative circle for metaphysicians to bounce between the two terms ontos and cosmos, and now we even find physicists and astronomers engaged in the same sort of semantic quest in determining which term is the more positive one in defining the other. We find it in their speculating about different levels of being and different levels of reality, and in speculating about the reality of possible worlds and in their speculating about the structure of the world. The basic question for them becomes whether ontos or comos is the prior term? But when we recognize that the interpretations we make of these models and systems of ultimate entities and their arrangement all have their origins in human artifice and that these conventionally constructed ontologies and cosmologies are very much like many of the verbal semantic games that we play. But however in the game of metaphysics there are no rules to play it as is the case with chess. I found in my experiences with the Metaphysical Society that there were no rules in doing metaphysics.

Chess unlike metaphysical games is a recognized sport defined by the International Olympic Committee. International chess competition is sanctioned by the Fédération Internationale des Échecs or World Chess Federation (FIDE). It is an international organization that connects the various national chess federations around the world and acts as the governing body of international chess competition. Chess is a rule governed game made up on earth and not in heaven. There is no chess then played in heaven! And given the nature and structure of the reality that is being sought there also are no philosophers are metaphysicians in heaven. As Whitehead joked, 'The pleasures of philosophy are denied to deity.'

We need to recognize that our ways of dealing with our problems with the world in everyday life are limited by the personal artificial frames of reference that we innovatively construct to deal with the problems of life. It is those things that we speak of in our everyday encounters with each other that are for the most part what we think of as real and fully by consensus are considered to be what is real for us. Noteworthy it is this ground in consensus about what is real that we use in our imaginations when we venture into the abstractions of formal systems. It is this consensus about common sense reality that we use to construct elements in our formal systems as we move away from everyday observations and to terms that we use to talk about the product of these speculations.

The simple descriptions we have of our ways of dealing with the world are for the most part basically simply rules of thumb that we use to guide us as we thread 
our ways through the games and frames that we create to manage the existential complexity of tensions and conflicts that we do not fully understand. These rules of thumb are just pragmatic ways that we use to live by. They are framed and interpreted and defined by the ordinary language in which we speak.

They are in turn limited in their uses by the constraints of our vernacular language in the ways that we have in using it. What I found striking in the work of Herbert Simon, Administrative Behavior: Study of the Decision-Making Processes in Administrative Organizations that in his treating organizational behavior at the time of his writing his first edition that the so called experts in speaking of 'administrative principles', so he maintained, there 'administrative principles' were very much like proverbs. To quote him he says:

It is a fatal defect of the current principles of administration that, like proverbs, they occur in pairs. For almost every principle one can find an equally plausible and acceptable contradictory principle. Although the two principles of the pair will lead to exactly opposite organizational recommendations, there is nothing in the theory to indicate which is the proper one to apply. To substantiate this criticism, it is necessary to examine briefly some of the leading principles. (1997: 29)

At this point in Simon's text, he goes on to examine four principles that occur in the literature of administration.

Proverbs are then very much like rules of thumb. They function much like algorithms in stating rules that we should act by in certain contexts and cultural situations. Like moral maxims they tell us what to do in practical actions. Whether we speak of actions or behaviors, they are in the ordinary ways of speaking very much the same thing. But note that the nominal difference of the use of the terms action and behavior as synonyms. This difference is extremely important to note in talking about human action. Especially this has been the case in the history of psychology where it has been unfortunate that Behaviorism especially that of the B. F. Skinner variety wants to reduce all human actions to human behaviors.

This behavioral way of describing human responses reduces human actions to external descriptions of how human beings act without any considerations about what is related to what is going on that is internal to human beings. Such a behavioral way of describing human actions rules out talk about actions, such as, those involving planning, making decisions, and actions described as thought or thinking. Such a description of human action in terms of behavior as found in Skinner consequently eschews all theory and reduces psychology to a model of inputs and outputs of black boxes. It ends up treating rewards and punishments in terms of reinforcement, positive or negative, of whatever it is that is going on in those black boxes with no intervening variables happily, loudly singing inner thoughts and feelings in between the inputs and outputs.

Simon in his work on administration is all about exposing the limitations of the decision making going on within an organization with a hierarchical structure of 
command and control wedded together with a rational and an efficient organization of tasks. His work is a study of the limitations of rational control and the limitations of communications that goes on between the various levels of such institutions as corporations or bureaucracies. But what is at issue in Simon's conception of public and private bureaucratic organizations is his realist ways of studying the structure and functions of these organizations. His conceptions of modeling are brought into question in his discussions of modeling in that he interprets these descriptions in terms of ordinary vernacular discourse that varies with different cultures, such as in the different cultures of business, government, science, and technology. He is confronted with the limitations of describing and interpreting the implications of the models that he brings to bear on his own discussion of the way he builds his own theory of administrative organizations.

These limitations in how we use different vernacular languages were recently discussed in an article in the New York Times by Guy Deutcher (2010), who maintains the linguistic thesis that the world looks different in other languages, and to express his point he quotes from the linguist, Roman Jakobson, the maxim, 'Languages differ essentially in what they must convey, and not in what they may convey.' And Deutcher goes on to say in the article, 'This maxim offers us the key to unlocking the real force of the mother tongue: if different languages influence our minds in different ways, this is not because of what our language allows us to think but rather because of what it habitually obliges us to think about.'

He illustrates his point by showing the constraints that gender has in certain languages of obliging us to reveal information about the sex of your companion. Or again he illustrates the different way a remote Australian aboriginal tongue, Guugu Yimithrirr, expresses orientation in small scale spaces that is built into their language not by left or right orientations, but by geographic directions based upon geographic directions such as north, east, south, and west. East is right, and west is left, and the two ways of using languages interfere in expressing orientations only when we have to check out the orientations in the aboriginal tongue when we reorient ourselves to these headings that had been determined by local map makers. Note in tropical latitudes east is where the sun rises. West is where the sun sets.

Much of the worldly wisdom about orientation we find to be over simplistic about the proper working conceptions that we have when our thinking confronts any new problematic complexity such as was found in the basic means and needs of the expertise that is needed in surveying. It is only by processes of simplification that we interpret the complexities of these complex models and the frames created by surveyors that developed much of our local talk about orientation of the local world that we think we know.

In my youth I lived along a street that I always thought ran north and south. It is only recently I put two and two together and recognized that a street farther to the north did not run parallel to it that was originally laid out on a north and south surveyed grid. When you fly over the Middle West the straight lines you see running 
supposedly north and south were laid out when they were first surveyed by magnetic compasses. Magnetic variation at the time of those original surveys was not truly north and south as would be the basis of a grid whose orientation towards the north is laid out or defined by its relation to the celestial sphere. Where I lived the first few streets were laid out parallel to the direction of the railroad tracks which was north by east.

And it is this simplicity of our ordinary ways of speaking that deceives us about the general application of some of our everyday rules of thumb, proverbs, aphorisms, and simple stories that we live by everyday. When we define technical language and the formal logical and mathematical systems and when we use them, we for the most part forget their origins in the language of everyday speech. In French Poststructuralist jargon the memory of those origins is spoken of as 'eclipsed'. There is a cultural forgetting of the origins of our sense and meaning of terms. It is that original source of meaning which is eclipsed. And too besides this forgetting, we need especially to recognize the pluralism of these multiple frames of reference and the inconsistencies that develop within these maxims or rules in our uses of them. They are in their expression context dependent and in their pluralism independent of the old contexts that were once used.

We are always limited in using conventional wisdom by the fact that our limited and finite perspective on complexity has been limited by the very biological, social, and political limits that constrain and restrict our wisdom. These limitations which constrain us have been introduced into the use of our vernacular languages at the time of the emergent origin of language in biological evolution of which we have no historical awareness. It was at the time of first language use and usage that we originally defined our human condition in our personal relationships to our culturally conditioned conceptions of space and time that have as a result immersed us in the consequent developments that evolved within our language.

But despite the sufficing utilities that we have in dealing with individual situations using general maxims, we need necessarily to expand and work on our difficulties and problems in using them. We need to develop different frames of conventional construction to articulate and calculate new solutions by maximizing and optimizing certain features in the models of logic, science, and math that we have developed to help us clarify and resolve our problems in using our practical maxims and rules, such as used in counting, ordering, and in determining orientation.

We learn to reinterpret our maxims and rules when we use our newly developed complex models in imaginative ways that are in a way reduced and reconstructed in much simpler terms in our using them. We learn to use mathematical and graphic models in ways that enable us to transcend our limitations in apprehending the complexity that occurs when we confine ourselves to the limits established by our ordinary ways of talking. We can by creating new models and new schemata together with new forms of language with new terms learn to rise above these bounded ordinary language limitations that we have in being situated as we are in our common 
everyday language usage, for that usage which we have come to understand is subject to evolutionary change when its inadequacy is recognized and exposed. It is at that point that our language use and usage moves on.

When we encounter these limits to our understanding in these models and schemata it is our imaginative use of them that helps us escape many of the inconsistencies to be found in our using what we deem only as having sufficing utilities and not optimal utilities. When our practical applications fail in their use we find then a need to revise them in much more precise and accurate ways. And we find in these revisions that we employ new modes of inquiry and new modes of disconfirmation. These revisions empirically allow us to progress in knowledge as creative creatures. They enable us to move beyond our cognitive limitations of being encased in the limits of our vernacular. We have learned in our use of such newly devised artificial frames that we have developed new ways to discover much better ways to find out how in the past we have been mistaken about the best way to resolve and solve our problems.

Formal schemata enable us to escape the limitations of memory, and they allow us to deal with complexity that overwhelms our normal apprehension of what we thought has happened, what is now happening, and is likely in certain determined ranges of probability likely to happen again. These newly developed models that we use to interpret our experiences are necessary to explore the complexity of our problematic situations that at times tends to overwhelm us.

These newly developed models are the foundation of all expertise. And we find in our interpretations of them that not only are these new models and visual schemata interpreted in many cases in figurative language, but they are often phrased in terms that when taken from literal lexical usage are fraught with multiple senses and imprecision. It is from these language limitations that we have as a result created many of the ambiguities that we find in the uses of terms. These ambiguities tend to confuse us in contexts that differ from those in which we originally learned to apply and use those terms. Such a dependence on models then presents the problem of recognizing how in formal languages that the terms that are used to generate these formal models are often open to various interpretations, which generate the paradoxes and contradictions that we find in our expanded uses of them in different contexts and situations.

The patterns and meanings of terms in formal systems are for the most part generated internally in the growth of our professional languages that are generated by technical definitions. But these technical terms are often reinterpreted and reenter into the vernacular in different ways. Just lately I was astonished as to how the hormone thyroxin as been transformed in my life time by organic chemistry. It is now no longer the simple hormone that my teachers once said it to be. We no longer can think of thyroxin in simpler terms and in the role that it supposedly played in the older models of interactions between it and other hormones. 
We especially find this breakdown between those who interpret these models for experimental purposes and those who create these models in formal and theoretical ways. There is a great divide between the language of experimental scientists and theoretical scientists about these matters. But what people in the end do not seem to recognize is that formal logical and mathematical languages are languages that enable us to exhibit formal complexity, but that they run into serious problems of interpretation when they are applied and used experimentally to test conjectures.

As a result of our human conditions and personal aims we consequently are bound within the limitations of our thinking. We are bound within the boundaries of short-term memory and the constraints of maintaining long term memory. And in addition we are bound within the perceptual limits of attention in our ordinary language use and usage in understanding our human situation as it relates and depends upon other people. We are caught with linguistic skills that have emerged from our biologically innate uses of language. We are embedded in social, cultural, and political conditions that frame our language perspectives. We are as individuals embedded in a life framed by personal choices and commitments. The net result is that we live in a world where our beliefs are questioned by our different uses of the multiple frames by which we relate to other people and how we relate to the culture that we find ourselves within.

Developments in mathematics, the sciences, and in technologies invert our conceptions of what is verbally simple. They have done so in the past as we have seen in history of sciences, such as in our revised conceptions of the movement of the sun, the moon, and the planets. They have revised our conceptions of space and time. The implications of such changing perspectives found in the models and schemata of the sciences gradually invert in time our conceptions of what is simple in ordinary everyday language and what is accepted in everyday common sense.

But the major error in using such modified models is to mistake their formal and informal imaginative structures as representations of the real world. Although we conceive and perceive them to be natural representations; yet existentially they are the projections of a linguistically defined emergent and a developing ego that seeks to escape its ignorance. My argument continues to be that the better perspective to take in thinking of the world is to think of models and visual schemata, not as naturalistic representations, but to look at the various patterns and sequences within them as artificial constructions devised from terms in our vernacular, which in the end frame for us what our personal conception of what the really real is in the end really like for us. But in the end reality is what is ordinarily thought real for us as we describe what we see happening about us and within us. It is what others can agree with us about in talking to us about what is really real for the both of us.

Human artifice is a development we find originating in the evolutionary development of what we think of as life. This raises all those questions raised about what is life? And since our conceptions of naturalistic processes is framed in artificially developed language and socially constructed schemata, it is a paradox to 
say just where life begins and emerges from physical and chemical processes that in the fields of biochemistry many purport to describe as externally real life processes. The important point in saying what knowledge is lies in the fact that we feel that we have a certain sense of what it is, but that certain sense of what it is actually is a product of an artificially created language that becomes a medium of exchange for us with others. All the models and schemata developed in math and science equally rest upon artificial creations that have been developed around the innate embedded artifices found in life processes that are emerging in the evolution of human life.

Life creates the emergent changing forms of life that in turn become sufficient for life to adjust and maintain its own form of life by replication. And the human brain especially was a product of that emergent change of new patterns developing in its anatomy for making adjustments. Our brains, as Krishnamurti maintained, are a product of many millennia of readjustments to maintain the species. It is not a machine searching for reality. Its forms and structures were created to maintain the human species. Language is a part of that emergent change of those survival seeking structures. But we as persons are not our brains. As Krishnamurti maintains, and this is the most important point about evolution, our brains belong to the emerging human species and not to us. We as creatures are actively going along with the ride. Evolution historically has been all about the species, that is, whatever it is we mean by what we call a species. A species is at least a name for some of those stabilities we see in the different kinds of life processes.

My argument is that the better perspective to take in thinking of the world is that instead of thinking of models and visual schematic as naturalistic representations, we should instead look upon these patterns and the sequences found in them as linguistic tools developed to exercise command and control. After all they are no more than artificial human constructions. We use them instrumentally as ways of constructing patterns of action in what we see as existential boundless complexity that we have in solving multiple tensions and conflicts. These guiding patterns of action have been generated by the emerging patterns in the brain or in our neural systems that have sufficed, especially in recent biological history in the prefrontal cortex to maintain the human species. But note this conception of the prefrontal cortex is again merely another simplified model of what is happening in this structure that we model and that we think of realistically as the brain. But where anatomically does a brain begin and end. How can we separate what we call a brain from the nervous system? Neurologically what defines a brain?

In interpreting these patterns as representation of fact and reality that is set forth in models and frames we should look upon our interpretations of them in the ordinary language that is used in the first place to shape and construct them. They are no more than artificial constructed frames that we use for the scaffolding our terms and concepts in our language. We use these schemata and frames in order to relate and to correlate our terms and concepts in thinking about the complexity of our lives and the complex problems we face in the world that seem most of the times beyond 
our rational capabilities. These models then are tools for the mind to think with and to use and to order and aggregate units of things into sets of things.

They are tools to sort out the patterns in the complexity of our thinking. We are severely limited in our ignorance about the facts of occurrence of things both past, present, and future. We are limited by the use of models that do not extend as modes of representation beyond the range of what we can study, as for example many of the micro-anatomical features that stretch beyond our abilities to image them we have no clear conception about. We are limited by not only by the linearity of our thinking, but we are limited in memory about what we think are the so called facts needed to cope with any understanding of our worlds.

And when we think about these artificial frames that we create, we should avoid projecting abstract terms and concepts that we use in different contexts and situations to say anything with certainty about them. We should recognize the limitations of our interpretation in our ways of looking at the world. These models as representation are nothing more than virtual or artificial organizations of experiences that enable us to remember and to recall. As we find in the paradoxes of philosophy, we are using these frames as representations of real structures in the world when in fact they are no more than scaffoldings for our languages that we mistake as being a true structural part of reality.

Our models and frames in the sciences were developed out of our seemingly innate senses of orientation and on our primitive ways of counting which in turn lay the foundations of mathematics. These models shape the way our languages have generated the roles we play in seeking solutions to questions that we believed that we were ignorant about. And when considered from the evolutionary point of view, they are to be found to be found in the very biology of our anatomy that shapes the geometry of our perception. And the net result of all of this is that we end up projecting these artificial constructions into our everyday psychological perceptual and inner experiences, and we end up thinking that they are the patterns to be found in the actual world.

The sum of it is that we are existentially situated in the frames of our ordinary language, which has been biologically and socially constructed. Biologically speaking our evolution has laid the linguistic foundation of our cognitive capabilities. The biological foundations of our language that were first used for making our social adjustments are to be found to exist in the human condition as an emerging social creature. The social interaction and mutual development of our cognitive abilities enable us as human creatures to become very creative creatures. In actuality the models and schemata that we find emerging from our basic innate frames are no more than additional tools and instruments of discovery that emerged out of an added number of new varieties of artifice created in evolution. They are in their creative development just new ways of saying what is normative or ideal in applying the terms of our language in our everyday practical experiences. But our idealism about these norms or ideals is shattered when they result in failure. 
What I want now to do in following chapters is to continue to expand in greater depth upon our modes of interpretations of models and frames, logical and mathematical, and to see how they always are interpreted and technically defined in much more complicated ways still using terms of ordinary language. We need to recognize that our different ways of dealing with our problems in dealing with the world are limited by these personal artificial frames of reference that we personally and innovatively construct in our everyday adjustments. And too we need to recognize in the pluralism of these multiple frames of reference the inconsistencies that develop within and between them the incompatible uses we find in our using them. We are always limited in using them by the fact that our limited and finite perspective on complexity is limited by the very biological, social, and political limits we have in using our languages.

But despite the sufficing utilities we use and with which we are able to calculate in using these different models, we can nevertheless in imaginative ways transcend their limitations by creating new models and new schema together with new language and new terms to rise above these bounded limitations. When we encounter these limits in our models and schemata it is our imaginative use of them that helps us escape many of the inconsistencies between them where their practical applications fail in the uses of them. These escapes from our limitations are rendered possible by our uses of the modes of disconfirmation that empirically allow us to progress in knowledge, thus empowering us as living creatures to get beyond our past incapacities.

Formal schemata enable us to escape the limitations of memory, and they allow us to deal with complexity that overwhelms our normal apprehension of what we thought has happened and what is now happening. They are thus necessary to explore the complexity that at times overwhelms us. Not only are models and visual schemata interpreted in many cases in figurative language, but they are often phrased in terms of literal lexical usage that are fraught with imprecision and ambiguities that have been created by the various changing senses of terms in the different contexts in which they are used. Such a dependence on models presents the cognitive problem of recognizing how in formal languages that the terms used to generate these formal models are often open to various interpretations, which in turn generate the paradoxes and contradictions that we find in them. Their formalization quite often is seen as other than what the interpreted defined meaning of the technical terms suggest.

What people in the end do not recognize is that formal logical and mathematical languages are languages that enable us to exhibit formal complexity by rules. They enable us to frame them in a language that allows us to axiomatize statements within them that make it possible for further changes in them that allow for new systems of logical implications to be explored that always have their own determinable logical consequences. We use them to map and to trace the complexity that we have to deal with when we are limited and constrained by ordinary ways of speaking.

In sum as a result of our human condition and our personal aims we are bound within the limitations of our thinking within the language usage that we agree upon. 
We are bound within the boundaries of short-term memory and the constraints of maintaining long term memory. And in addition we are bound within the perceptual limits of attention in our uses of ordinary language usage in the understanding of our human situation as it relates and depends upon other people. We are caught with linguistic skills that have emerged from our biologically innate uses of language. We are embedded in social, cultural, and political conditions that frame our language perspectives. We as individuals are embedded in a life framed by personal choices and commitments.

As Herbert Simon suggests we need to look at our communications to see what goes on between us. To me such a suggestion recommends my own outlook that we need to look at the role rhetoric plays in communication very carefully, especially as we use rhetoric in the sciences and technologies to reach cognitive mutual understanding about what to us appears real and functional in our ways of doing things in furthering what is important in our lives. 